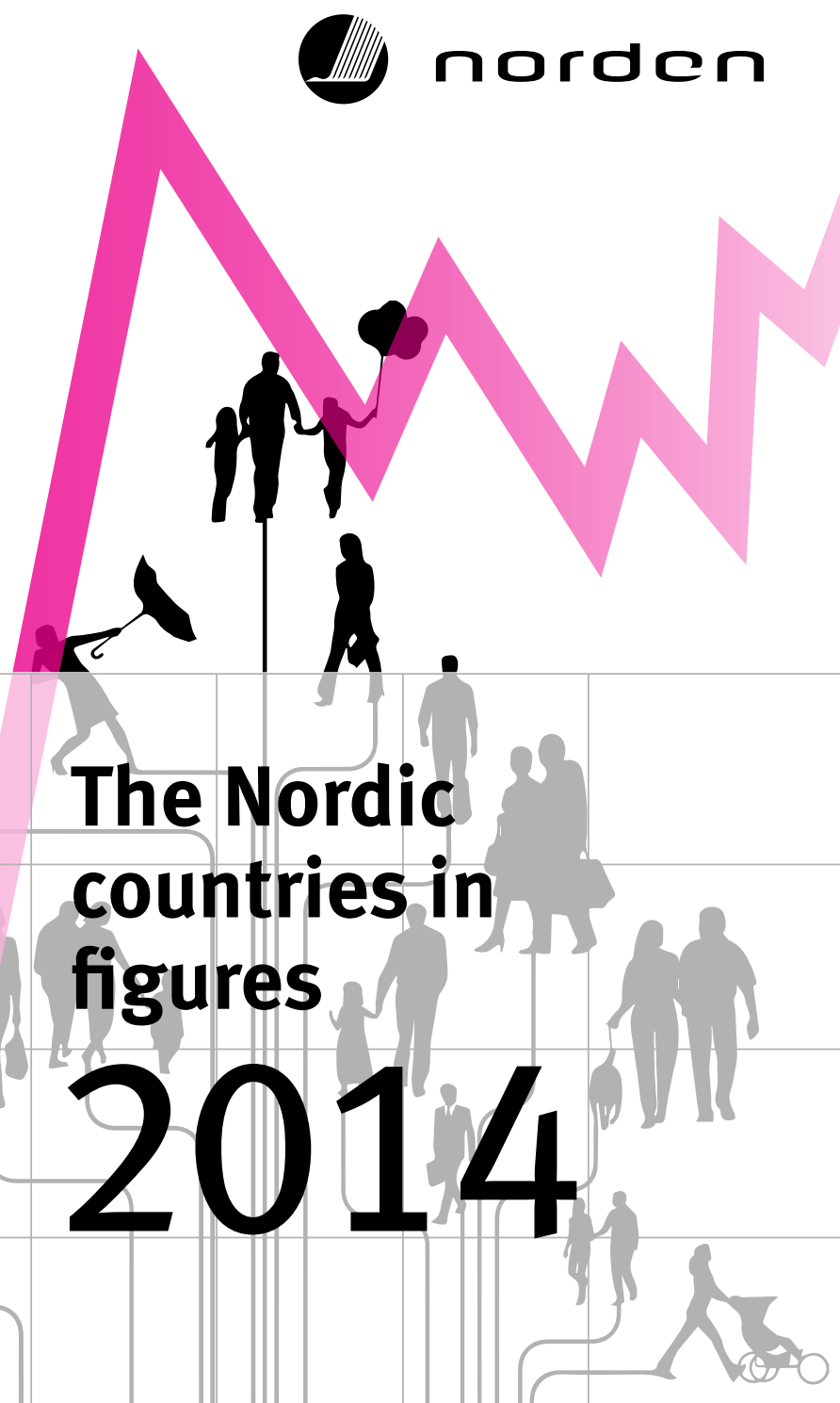


Order your Nordic Statistical Yearbook 2014 on www.norden.org/nordpub 
Nordic countries in figures 2014

ANP 2014:752 


\section{Nordic countries in figures 2014}

ISBN 978-92-893-3796-0 (PRINT)

ISBN 978-92-893-3818-9 (PDF)

http://dx.doi.org/10.6027/ANP2014-752

ANP 2014:752

(C) Nordic Council of Ministers 2014

Cover design: Jette Koefoed/Signelements

Photos p 4-5: ( ) Nordic Council of Ministers

Print: Rosendahls-Schultz Grafisk A/S

Copies: 1000

Printed in Denmark

Main suppliers of data: The Nordic national statistical institutes

Ed: Klaus Munch Haagensen, Statistics Denmark

Database: Troels A. Vestergaard, Statistics Denmark

\section{Nordic co-operation}

Nordic co-operation is one of the world's most extensive forms of regional collaboration, involving Denmark, Finland, Iceland, Norway, Sweden, and the Faroe Islands, Greenland, and Åland.

Nordic co-operation has firm traditions in politics, the economy, and culture. It plays an important role in European and international collaboration, and aims at creating a strong Nordic community in a strong Europe.

Nordic co-operation seeks to safeguard Nordic and regional interests and principles in the global community. Common Nordic values help the region solidify its position as one of the world's most innovative and competitive.

\section{Nordic Council of Ministers}

Ved Stranden 18

DK-1061 Copenhagen K

Tel: +4533960200

www.norden.org 


\section{Contents}

The Nordic countries

Key figures

Geography and climate

Environment and energy

Population

Health

Social integration

Education
$4 \quad$ Labour market $\quad 18$

6 Elections 20

8 Culture 21

9 The Economy 22

10 Foreign trade 25

13 Public finance and prices 27

$15 \quad$ Science and technology 30

The symbol $\square$ and the reference that appears below a table or diagram identifies the table in the databank that has served as its source. All the tables may be found on the database. The reference after the pc-symbol also works as a link to the relevant database-table. With a click on the reference you get directly into the table in question.

Throughout the book, the following symbols and country codes are used:

Symbols

Nil

Less than half

Not applicable

Data not available
Country codes

Denmark: DK

Faroe Islands: FO

Greenland: GL

Finland: FI

Euro area: EA17
Åland: AX

Iceland: IS

Norway: NO

Sweden: SE

The EU: EU27

To describe the different countries in the figures, the following colours are used:

Denmark: Greenland: Åland: Norway:

Faroe Islands: Finland: — Iceland: — Sweden:

Euro area: The EU: 


\section{The Nordic countries}
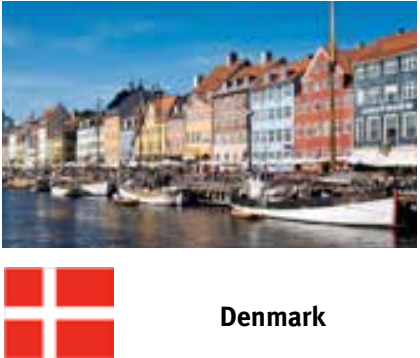

Area $\mathrm{km}^{2}$

Population

Status/Form

of government

Head of state

Copenhagen
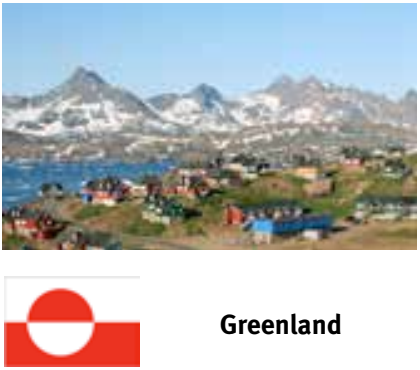

Area $\mathrm{km}^{2}$

Population

Status/Form

of government

Head of state

Nuuk

\section{Denmark}

43561

5627235

Constitutional

monarchy

Queen Margrethe II

1246611
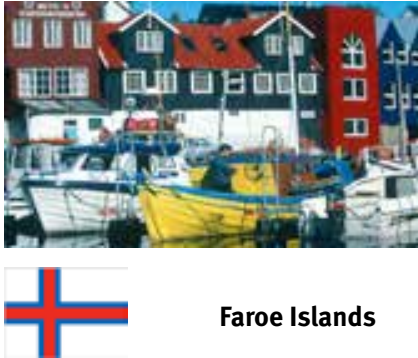

Area $\mathrm{km}^{2}$

Population

Status/Form

of government

Head of state

Tórshavn

\section{Faroe Islands}

1396

48228

Home rule - within the Kingdom of

Denmark

Queen Margrethe II 19948
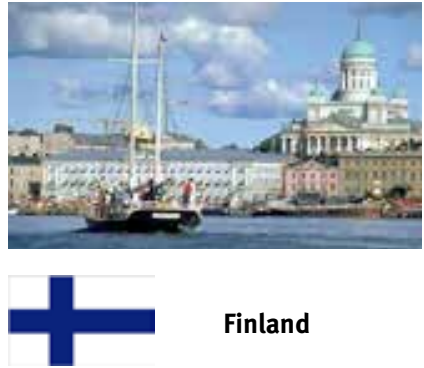

Finland

Area $\mathrm{km}^{2}$

338432

Population

5451270

of government

Head of state
Status/Form

Denmark

Queen Margrethe II 16818

Self government within the Kingdom of Head of state

Helsinki
Republic

President

Sauli Niinistö

1090616 

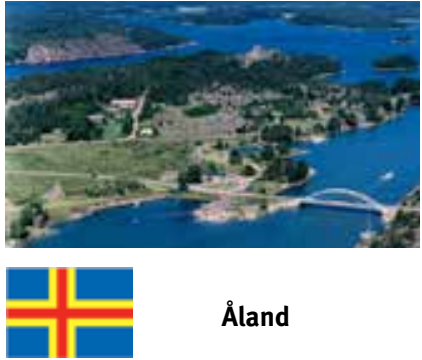

Area $\mathrm{km}^{2}$

Population

Status/Form

of government

Head of state

Mariehamn
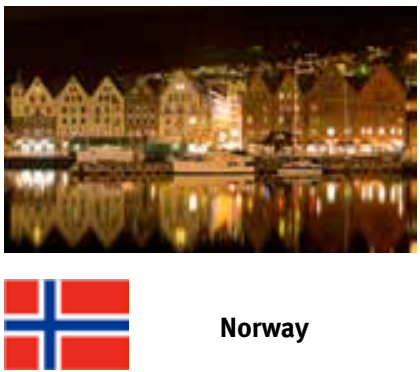

Area $\mathrm{km}^{2}$

Population

Status/Form

of government

Head of state

Oslo

\section{Åland}

1580

28666

Home rule - within the Republic of

Finland

President

Sauli Niinistö

11393

Norway

323771

5109056

Constitutional

monarchy

King Harald V

1210220
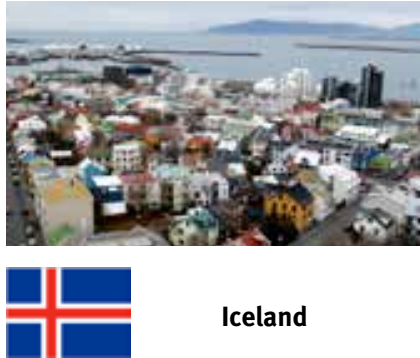

Iceland

Area $\mathrm{km}^{2}$

103492

Population

325671

Status/Form

of government

Republic

Head of state

President Ólafur

Ragnar Grímsson

Reykjavik

208752
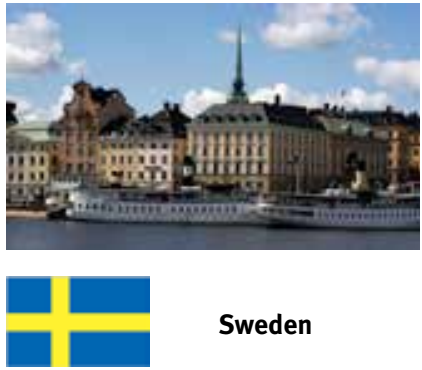

\section{Sweden}

Area $\mathrm{km}^{2}$

447420

Population

9644864

Status/Form

Constitutional

of government

monarchy

Head of state

King Carl XVI Gustaf

Stockholm

2163042 


\section{Key figures}

The Nordic countries in an international context - key figures $2013^{1}$

\begin{tabular}{|c|c|c|}
\hline $\begin{array}{r}\text { Nordic } \\
\text { countries }\end{array}$ & EU & Japan \\
\hline
\end{tabular}

Total land area,

$1000 \mathrm{sq} \mathrm{km}$

1147

4236

378

9832

Arable land, per cent

of land area

7.3

28.4

12.5

17.5

Forest area, per cent

of land area

56.1

37.7

68.6

33.3

Population, million

26

$486 \quad 128$

309

Density

(inhabitants $/ \mathrm{km}^{2}$ )

$17 \quad 119$

338

32

Total fertility rate

1.8

1.6

1.4

1.9

Life expectancy, years

\begin{tabular}{lrrrr} 
men & 79.9 & 77.5 & 79.9 & 76.3 \\
women & 83.9 & 83.1 & 86.4 & 81.1 \\
\hline Unemployment rate & 7.1 & 10.9 & 4.0 & 7.4 \\
$\quad$ men & 7.3 & 10.9 & 4.3 & 7.6 \\
women & 6.8 & 10.9 & 3.7 & 7.1 \\
\hline $\begin{array}{l}\text { GDP, PPS (million US\$) } \\
\text { GDP per capita, }\end{array}$ & 1675 & 13069 & 4792 & 17058 \\
$\begin{array}{l}\text { PPS (US\$) } \\
\text { Tax revenue as }\end{array}$ & 47513 & 34083 & 37630 & 53960 \\
$\begin{array}{l}\text { percentage of GDP } \\
\begin{array}{l}\text { Tax revenue per } \\
\text { capita (US\$) }\end{array}\end{array}$ & 44.9 & 40.7 & 28.3 & 26.9 \\
\hline $\begin{array}{l}\text { Total imports C.I.F } \\
\text { (billion US\$) }\end{array}$ & 27416 & 10469 & 13308 & 12599 \\
$\begin{array}{l}\text { Total exports F.O.B } \\
\text { (billion US\$) }\end{array}$ & 437 & 5882 & 886 & 2240 \\
$\begin{array}{l}\text { Trade balance } \\
\text { (billion US\$) }\end{array}$ & 534 & 6063 & 799 & 1579 \\
\hline
\end{tabular}

${ }^{1} 2013$ or latest available data. The Nordic countries in this table consist of Denmark, Finland, Iceland, Norway and Sweden. EU: The 27 or 28 member states of the European Union as per 1 January 2013 or 1 January 2014. PPS: Purchasing Power Standards which eliminates the differences in price levels between the countries. 


\section{Area (ice-free)}

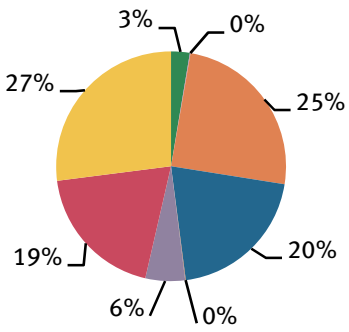

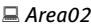

\section{Population}

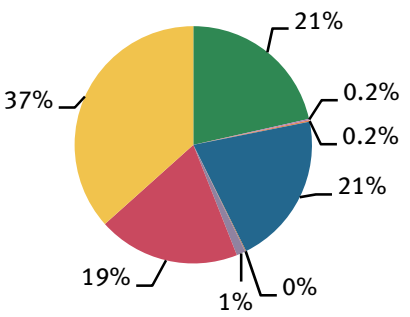

- Denmark 21\%

- Faroe Islands $<1 \%$

- Greenland < 1\%

- Finland 21\%

Åland < $1 \%$

- Iceland 1\%

- Norway $19 \%$

Sweden 37\%

Рopu01

\section{Gross national income}

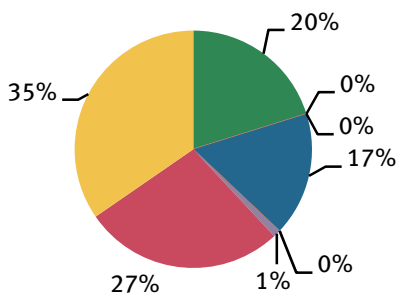

Denmark $20 \%$

- Faroe Islands $<1 \%$

Greenland < $1 \%$

- Finland $17 \%$

- Åland $<1 \%$

- Iceland 1\%

norway $27 \%$

Sweden 35\% 
Geography and climate

\section{Land use 2013}

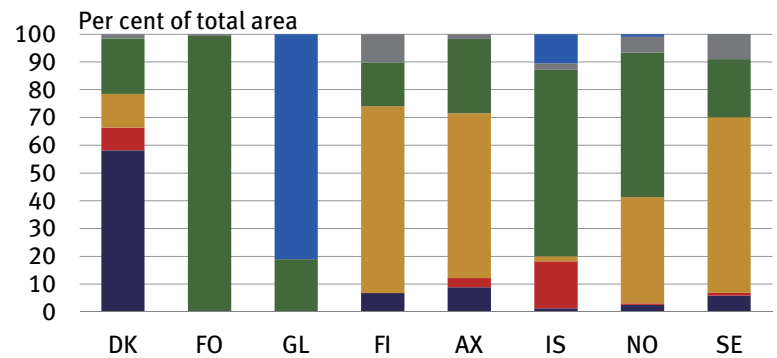

- Arable land and gardens

- Meadows and pastures

- Forests

- Other land area

- Lakes

- Icecap

Area02

\section{Average temperature in the Nordic capitals}

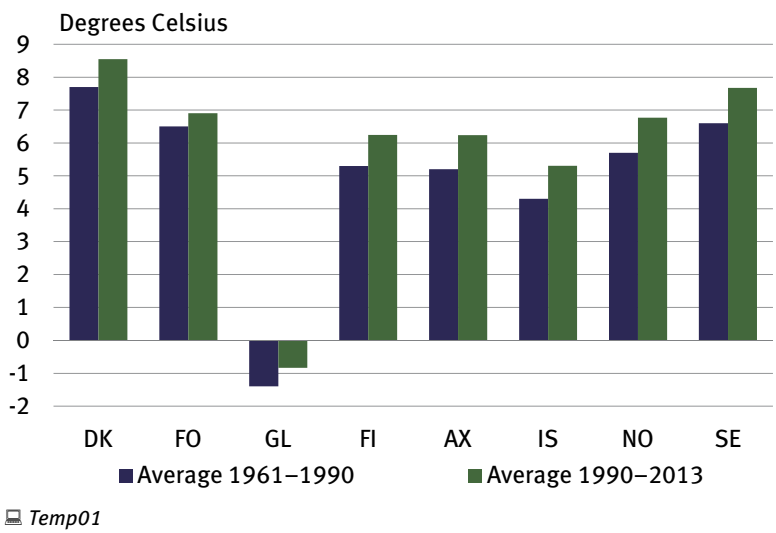


Greenhouse gas emissions per capita

\begin{tabular}{lrrrrr} 
& DK & FI & IS & NO & SE \\
\hline $\mathrm{CO}_{2}$-equivalents, tonnes & & & & & \\
\hline 1990 & 13.6 & 14.1 & 13.7 & 11.7 & 8.5 \\
1995 & 14.8 & 13.8 & 12.2 & 11.4 & 8.4 \\
2000 & 13.0 & 13.4 & 13.7 & 11.9 & 7.8 \\
2005 & 12.0 & 13.1 & 12.9 & 11.6 & 7.5 \\
2012 & 9.4 & 11.3 & 13.9 & 10.5 & 6.1 \\
\hline
\end{tabular}

Emis 11 and Popu02

\section{Indigenous energy production 2012}

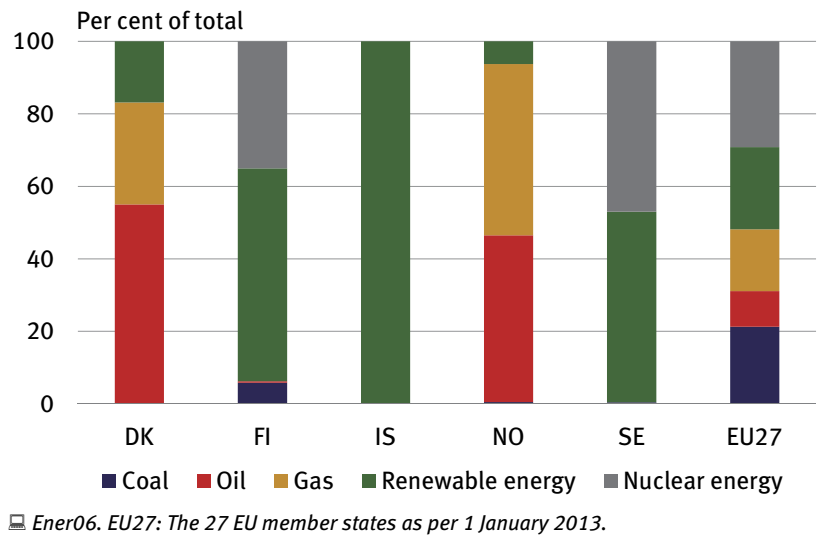




\section{Population}

Nordic population 1 January 2014

\section{Total}

The Nordic

\section{countries, total}

Denmark

Faroe Islands

Greenland

Finland

Åland

Iceland

Norway

Sweden

26262575
5627235
48197
56282
5451270
28666
325671
5109056
9644864

Men

Women

Popu01. Finland includes Åland.

Population trends according to the latest population projections

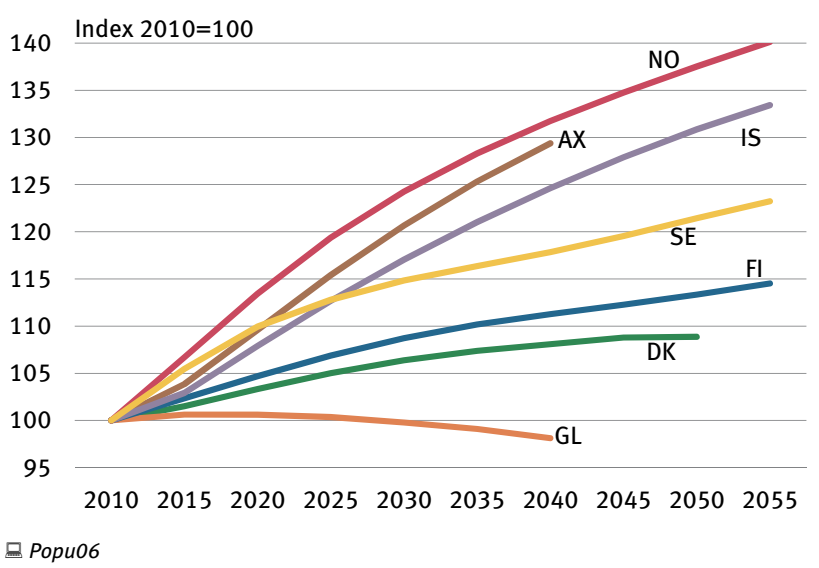


Total fertility rate

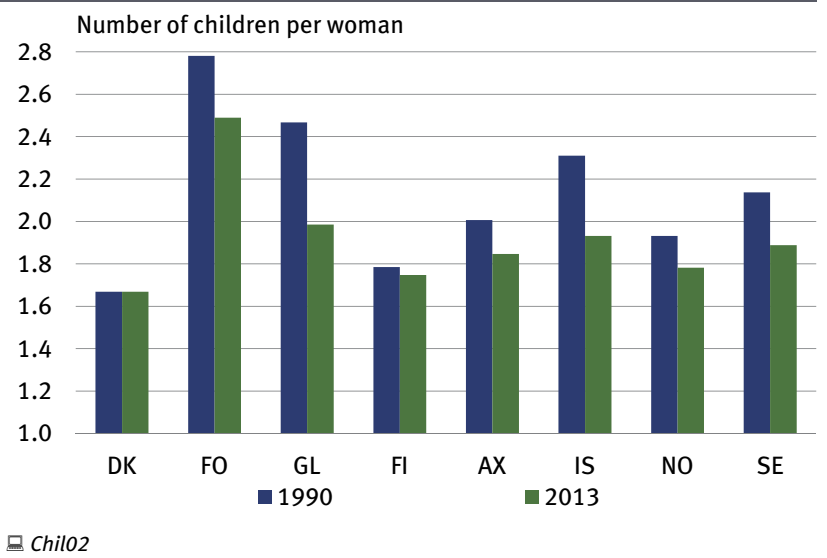

Life expectancy for a newborn baby, years

\begin{tabular}{lcccccccc} 
& DK & FO & GL & FI & AX & IS & NO & SE \\
\hline Men & & & & & & & & \\
\hline 1990 & 72.0 & 73.1 & 60.7 & 70.9 & 74.7 & 75.0 & 73.4 & 74.8 \\
1995 & 72.6 & 74.5 & 62.3 & 72.8 & 75.1 & 76.5 & 74.8 & 76.2 \\
2000 & 74.3 & 76.2 & 62.8 & 74.1 & 78.5 & 77.6 & 76.0 & 77.4 \\
2005 & 75.6 & 77.1 & 65.5 & 75.5 & 77.6 & 79.2 & 77.7 & 78.4 \\
2010 & 77.1 & 78.8 & 67.9 & 76.7 & 81.2 & 79.7 & 78.9 & 79.5 \\
2013 & 78.0 & 79.3 & 68.7 & 77.8 & 79.7 & 80.8 & 79.3 & 80.1 \\
& & & & & & & & \\
Women & & & & & & & & \\
1990 & 77.7 & 80.3 & 68.4 & 78.9 & 82.8 & 80.1 & 79.8 & 80.4 \\
1995 & 77.8 & 81.3 & 68.3 & 80.2 & 81.1 & 80.6 & 80.8 & 81.5 \\
2000 & 79.0 & 81.6 & 68.0 & 81.0 & 81.5 & 81.4 & 81.4 & 82.0 \\
2005 & 80.2 & 82.1 & 70.9 & 82.3 & 83.6 & 83.1 & 82.5 & 82.8 \\
2010 & 81.2 & 83.9 & 72.9 & 83.2 & 84.2 & 83.7 & 83.2 & 83.5 \\
2013 & 81.9 & 83.4 & 73.5 & 83.8 & 83.2 & 83.7 & 83.6 & 83.7 \\
\hline
\end{tabular}

마fe01 


\section{Population}

\section{Asylum requests}

\begin{tabular}{rrrrrrr} 
& DK & FI & IS & NO & SE & Total \\
\hline 1990 & 5292 &.. & 4 &.. & 29420 &.. \\
1995 & 5104 &.. & 4 & 1460 & 9047 &.. \\
2000 & 10347 & 3170 & 25 & 10843 & 16303 & 40688 \\
2005 & 1283 & 3574 & 87 & 5402 & 17530 & 27876 \\
2006 & 922 & 2324 & 39 & 5320 & 24322 & 32927 \\
2007 & 1029 & 1505 & 42 & 6528 & 36207 & 45311 \\
2008 & 951 & 4035 & 73 & 14431 & 24353 & 43843 \\
2009 & 2022 & 5988 & 34 & 17226 & 24194 & 49464 \\
2010 & 2844 & 4018 & 44 & 10064 & 31819 & 48789 \\
2011 & 3573 & 3087 & 75 & 9053 & 29670 & 45458 \\
2013 & 5107 & 3238 & 173 & 11983 & 54264 & 74765
\end{tabular}

모인

\section{Net migrations}

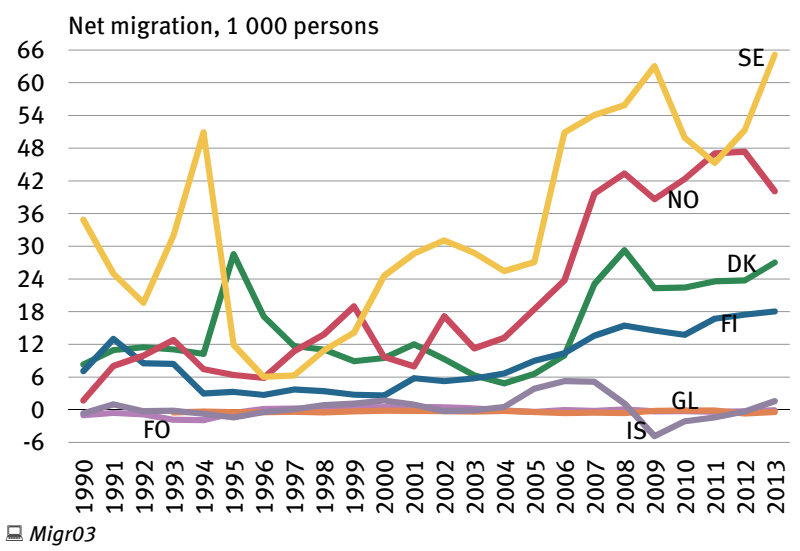


Health

Non-daily smokers

$\begin{array}{llllll}\text { DK } & \text { FO } & \text { FI } & \text { IS } & \text { NO } & \text { SE }\end{array}$

Percent

Men

\begin{tabular}{rrrrrrr}
\hline 1995 & 56 &.. & 71 & 68 & 67 & 78 \\
2000 & 69 &.. & 73 & 77 & 69 & 83 \\
2005 & 72 & 72 & 74 & 80 & 73 & 86 \\
2010 & 77 &.. & 77 & 85 & 81 & 87 \\
2011 &.. & 73 & 78 & 86 & 84 &.. \\
2012 & 83 & 73 & 79 & 85 & 85 &..
\end{tabular}

Women

\begin{tabular}{rrrrrrr}
\hline 1995 & 61 &.. & 81 & 67 & 68 & 76 \\
2000 & 74 &.. & 80 & 78 & 68 & 79 \\
2005 & 77 & 73 & 82 & 80 & 76 & 82 \\
2010 & 81 &.. & 84 & 86 & 81 & 85 \\
2011 &.. & 75 & 85 & 86 & 84 &.. \\
2012 & 83 & 72 & 82 & 87 & 86 &.. \\
\hline
\end{tabular}

Heal01

Sale of alcoholic beverages

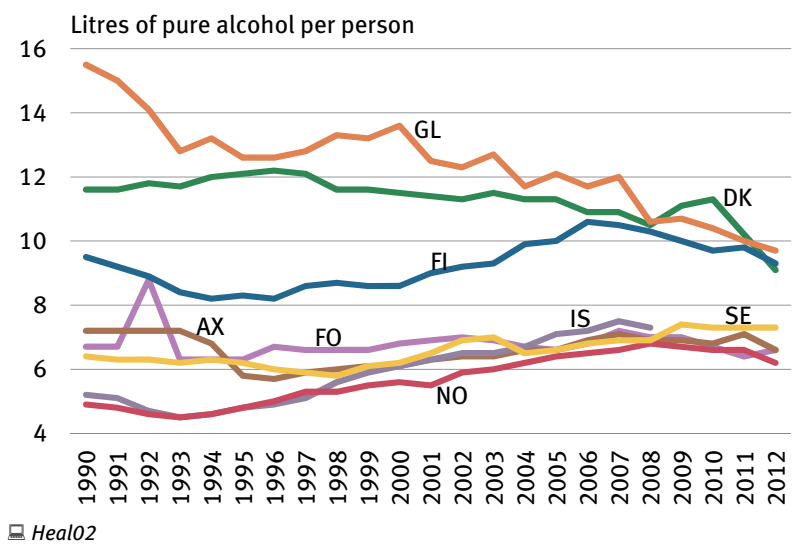


Health

Causes of death per 100000 people

\begin{tabular}{|c|c|c|c|c|c|c|c|c|}
\hline & DK & FO & GL & FI & $A X$ & IS & NO & SE \\
\hline \multicolumn{9}{|l|}{ Men } \\
\hline \multicolumn{9}{|l|}{2005} \\
\hline Cancer & 300 & 206 & 218 & 215 & 310 & 193 & 224 & 256 \\
\hline $\begin{array}{l}\text { Cardiovascu- } \\
\text { lar diseases }\end{array}$ & 303 & 335 & 185 & 364 & 338 & 226 & 300 & 418 \\
\hline Suicide & 17 & 12 & 89 & 28 & 23 & 16 & 16 & 19 \\
\hline Accidents & 35 & 37 & 82 & 81 & 43 & 26 & 50 & 44 \\
\hline \multicolumn{9}{|l|}{2012} \\
\hline Cancer & 287 & 182 & 208 & 232 & 279 & 192 & 229 & 242 \\
\hline $\begin{array}{l}\text { Cardiovascu- } \\
\text { lar diseases }\end{array}$ & 230 & 261 & 131 & 382 & 332 & 236 & 238 & 350 \\
\hline Suicide & 16 & .. & 100 & 25 & 12 & 18 & 15 & 17 \\
\hline Accidents & 24 & 63 & 60 & 63 & 61 & 25 & 39 & 36 \\
\hline \multicolumn{9}{|l|}{ Women } \\
\hline \multicolumn{9}{|l|}{2005} \\
\hline Cancer & 276 & 212 & 225 & 189 & 198 & 152 & 213 & 227 \\
\hline $\begin{array}{l}\text { Cardiovascu- } \\
\text { lar diseases }\end{array}$ & 322 & 334 & 188 & 389 & 455 & 217 & 328 & 436 \\
\hline Suicide & 6 & 1 & 86 & 10 & 5 & 6 & 7 & 8 \\
\hline Accidents & 27 & 19 & 53 & 36 & 17 & 18 & 34 & 31 \\
\hline \multicolumn{9}{|l|}{2012} \\
\hline Cancer & 256 & 201 & 227 & 200 & 242 & 160 & 203 & 218 \\
\hline $\begin{array}{l}\text { Cardiovascu- } \\
\text { lar diseases }\end{array}$ & 239 & 244 & 136 & 382 & 400 & 221 & 281 & 384 \\
\hline Suicide & 5 & .. & 27 & 8 & 7 & 4 & 6 & 7 \\
\hline Accidents & 16 & 13 & 38 & 33 & 21 & 16 & 34 & 25 \\
\hline
\end{tabular}


Children in day care by age 2013

\begin{tabular}{|c|c|c|c|c|c|c|c|}
\hline & DK & FO & $\mathbf{F I}$ & $A X$ & IS & NO & SE \\
\hline \multicolumn{8}{|c|}{ Per cent of age group } \\
\hline 0 years & 18.9 & 18.0 & 0.8 & 0.7 & 5.7 & 3.8 & 0.0 \\
\hline 1 years & 88.5 & 83.4 & 28.7 & 28.9 & 73.8 & 69.0 & 50.9 \\
\hline 2 years & 92.0 & 92.0 & 52.2 & 63.5 & 94.9 & 90.3 & 91.9 \\
\hline 3 years & 97.1 & 88.0 & 68.3 & 88.3 & 96.3 & 96.5 & 96.2 \\
\hline 4 years & 96.3 & 99.4 & 74.0 & 93.4 & 96.8 & 98.8 & 97.5 \\
\hline 5 years & 97.7 & 93.0 & 78.4 & 95.9 & 93.7 & 97.7 & 97.6 \\
\hline
\end{tabular}

Chil03. Children in fully or partly publicly funded day care. NO: 2012 data.

\section{Maternity and paternity day benefits 2013}

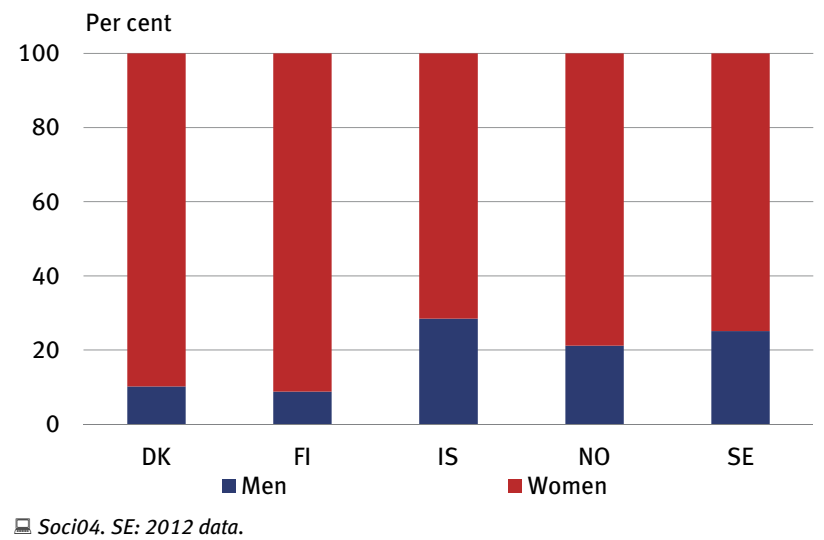




\begin{tabular}{ll|lr|ll} 
Reading literacy & & Mathematical literacy & Scientific literacy & \\
\hline Japan & 538 & Korea, Rep. of & 554 & Japan & 547 \\
Korea, Rep. of & 536 & Japan & 536 & Finland & 545 \\
\hline Finland & 524 & Switzerland & 531 & Estonia & 541 \\
\hline Canada & 523 & Netherlands & 523 & Korea, Rep. of & 538 \\
Ireland & 523 & Estonia & 521 & Poland & 526 \\
Poland & 518 & Finland & 519 & Canada & 525 \\
Estonia & 516 & Canada & 518 & Germany & 524 \\
New Zealand & 512 & Poland & 518 & Netherlands & 522 \\
Australia & 512 & Belgium & 515 & Ireland & 522 \\
Netherlands & 511 & Germany & 514 & Australia & 521 \\
Belgium & 509 & Austria & 506 & New Zealand & 516 \\
Switzerland & 509 & Australia & 504 & Switzerland & 515 \\
Germany & 508 & Slovenia & 501 & United Kingdom & 514 \\
France & 505 & Ireland & 501 & Slovenia & 514 \\
Norway & 504 & New Zealand & 500 & Czech Republic & 508 \\
United Kingdom & 499 & Denmark & 500 & Austria & 506 \\
United States & 498 & Czech Republic & 499 & Belgium & 505 \\
\hline Denmark & 496 & France & 495 & OECD average & 501 \\
\hline OECD average & 496 & OECD average & 494 & France & 499 \\
\hline Czech Republic & 493 & United Kingdom & 494 & Denmark & 498 \\
Italy & 490 & Iceland & 493 & United States & 497 \\
Austria & 490 & Luxembourg & 490 & Spain & 496 \\
Hungary & 488 & Norway & 489 & Norway & 495 \\
Portugal & 488 & Portugal & 487 & Hungary & 494 \\
Spain & 488 & Italy & 485 & Italy & 494 \\
Luxembourg & 488 & Spain & 484 & Luxembourg & 491 \\
Israel & 486 & Slovak Republic & 482 & Portugal & 489 \\
Iceland & 483 & United States & 481 & Sweden & 485 \\
Sweden & 483 & Sweden & 478 & Iceland & 478 \\
\hline Slovenia & 481 & Hungary & 477 & Slovak Republic & 471 \\
Greece & 477 & Israel & 466 & Israel & 470 \\
Turkey & 475 & Greece & 453 & Greece & 467 \\
Slovak Republic & 463 & Turkey & 448 & Turkey & 463 \\
Chile & 441 & Chile & 423 & Chile & 445 \\
Mexico & 424 & Mexico & 413 & Mexico & 415 \\
\hline & & & & \\
PIsad & & & & \\
\hline
\end{tabular}




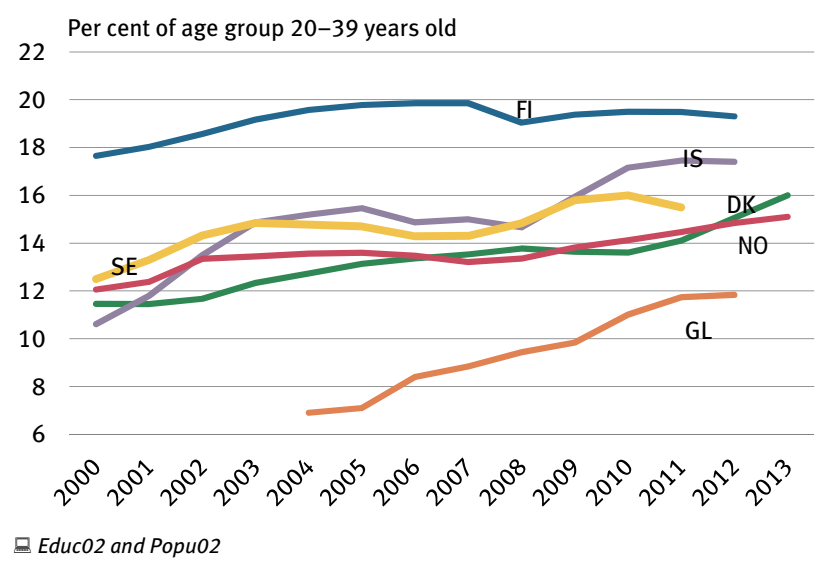

Nordic students abroad by destination 2012/2013

\begin{tabular}{lrrrrrr}
\hline Persons from & DK & FI & AX & IS & NO & SE \\
Total & $\mathbf{3 8 7 6}$ & $\mathbf{6 2 5 6}$ & $\mathbf{1 0 6 8}$ & $\mathbf{2 3 3 3}$ & $\mathbf{1 5} 746$ & $\mathbf{2 2} \mathbf{3 1 8}$ \\
Percent of total & & & & & & \\
Country of study & & & & & & \\
Nordic total & 21.0 & 21.8 & 96.4 & 46.7 & 21.3 & 13.1 \\
Denmark &. & 2.3 & 0.6 & 32.7 & 16.7 & 8.1 \\
Finland & 0.2 &. & 27.0 & 0.1 & 0.1 & 1.1 \\
Iceland & 1.2 & 0.2 & 0.0 &. & 0.2 & 0.2 \\
Norway & 6.1 & 0.9 & 0.9 & 3.1 &. & 3.8 \\
Sweden & 13.5 & 18.4 & 68.0 & 10.8 & 4.3 &. \\
& & & & & & \\
France & 1.8 & 1.9 & 0.1 & 1.2 & 1.3 & 3.5 \\
Germany & 6.2 & 4.2 & 0.1 & 3.2 & 1.3 & 3.3 \\
Great Britain & 39.3 & 29.9 & 1.0 & 10.8 & 29.9 & 21.1 \\
USA & 9.3 & 6.3 & 0.5 & 15.2 & 10.8 & 22.0 \\
Other & 22.4 & 36.0 & 1.9 & 22.9 & 35.4 & 36.9 \\
\hline Educ05 & & & & & &
\end{tabular}


Activity rates by sex 2013

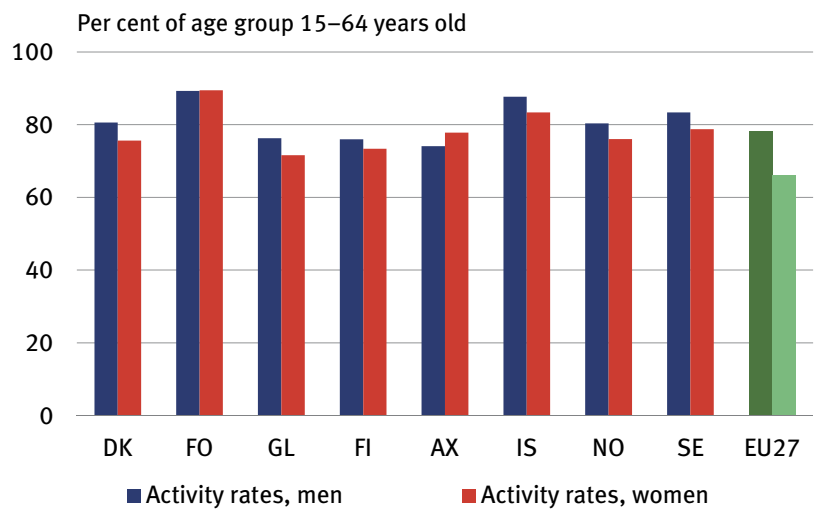

Work02. EU27: The 27 EU member states as per 1 January 2013.

\section{Unemployment}

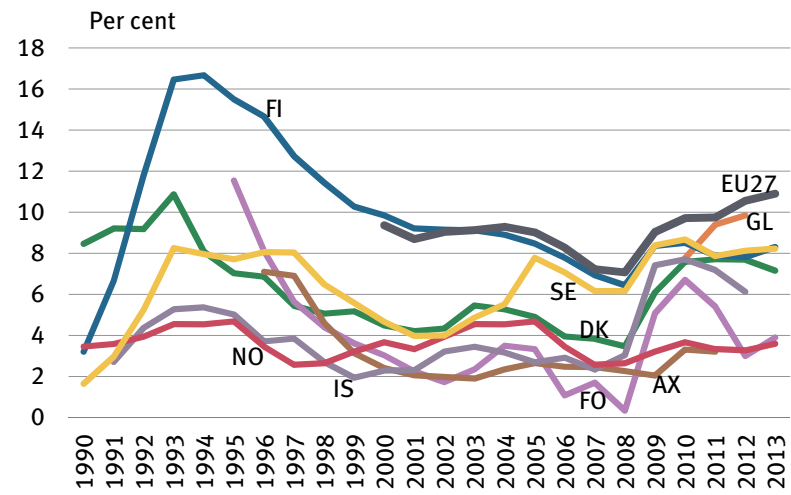

Key01. EU-harmonized unemployment figures except Faroe Islands and Åland. EU27: The 27 EU member states as per 1 January 2013. 
Unemployment by age 2013

$\begin{array}{llllllll}\text { DK } & \text { FO } & \text { GL } & \text { FI } & \text { AX } & \text { IS } & \text { NO } & \text { SE }\end{array}$

Per cent of labour force

\begin{tabular}{lrrrrrrrr} 
Total & $\mathbf{7 . 2}$ & $\mathbf{3 . 9}$ & $\mathbf{9 . 8}$ & $\mathbf{8 . 3}$ & $\mathbf{3 . 2}$ & $\mathbf{6 . 1}$ & $\mathbf{3 . 6}$ & $\mathbf{8 . 2}$ \\
$16-19$ & 15.8 & 15.4 & 20.7 & 29.6 & 11.8 & 16.2 & 12.3 & 36.7 \\
$20-24$ & 11.1 & 4.5 & 16.5 & 15.8 & 6.8 & 11.7 & 7.5 & 18.7 \\
$25-34$ & 8.6 & 3.1 & 10.8 & 7.9 & 3.2 & 6.6 & 4.2 & 8.1 \\
$35-44$ & 5.5 & 2.0 & 8.2 & 6.1 & 1.8 & 3.6 & 2.7 & 5.4 \\
$45-54$ & 5.2 & 2.4 & 8.3 & 5.9 & 3.0 & 3.6 & 2.0 & 4.9 \\
$55-64$ & 5.1 & 3.1 & 6.9 & 7.0 & 3.1 & 4.3 & 1.3 & 5.1 \\
\hline
\end{tabular}

@Work02

Part time employment by sex 2013

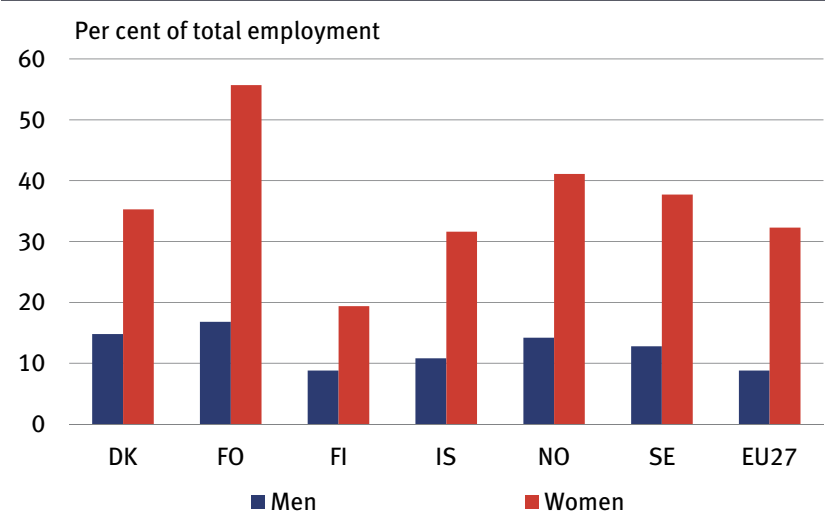

므 Labo03. EU27: The 27 EU member states as per 1 January 2013. 
Elections

Valid votes at the last two national elections

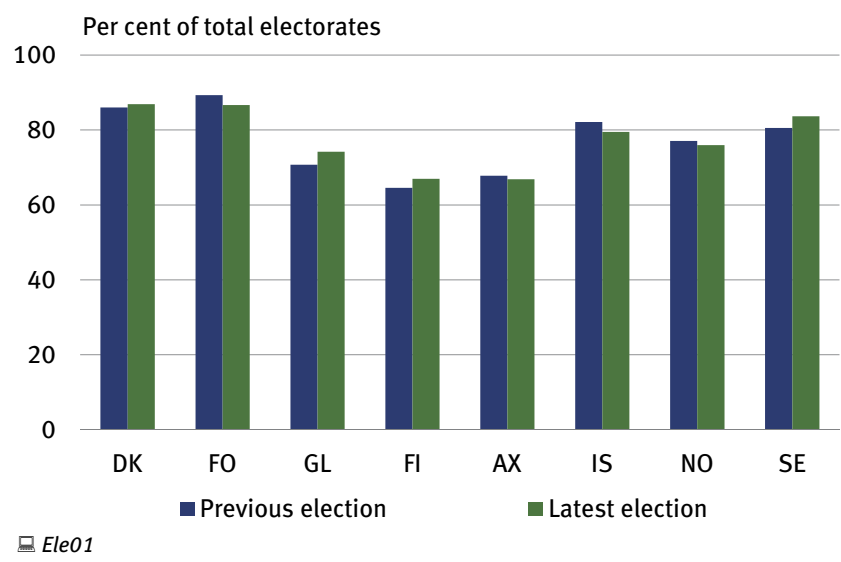

Candidates elected to national parliaments, latest election

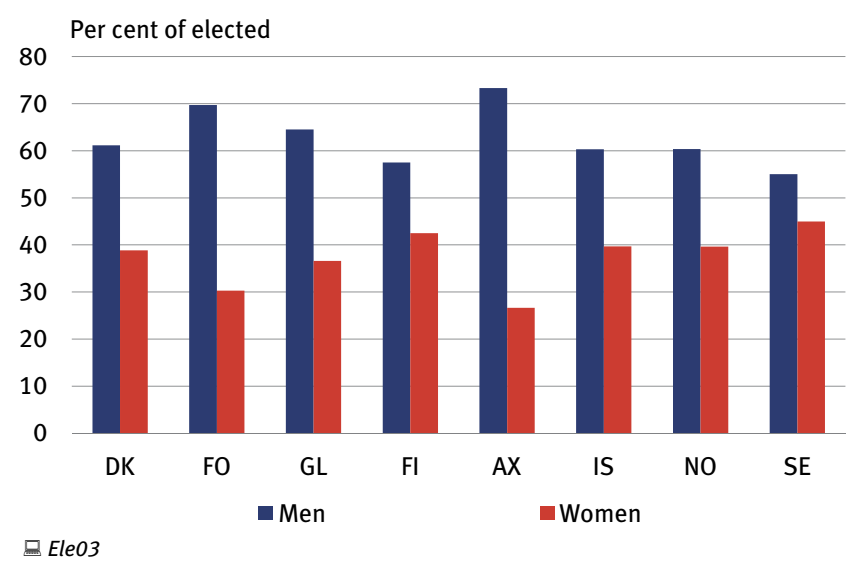


Culture

Sale of music 2011

\begin{tabular}{lrrrrr} 
& DK & FI & IS & NO & SE \\
\hline $\begin{array}{lrrrr}\text { 1000 euro } \\
\text { Total sale }\end{array}$ & 56451 & 33139 & 4206 & 64911 & $\mathbf{9 2 9 8 3}$ \\
Digital sale & 22043 & 8174 & 124 & 17883 & 47568 \\
$\begin{array}{l}\text { Percent } \\
\text { Total sale }\end{array}$ & 100 & 100 & 100 & 100 & 100 \\
Digital sale & 39.1 & 24.7 & 3.0 & 27.6 & 51.2 \\
\hline
\end{tabular}

몬19

Number of visits to museums 2013

Visits per person

5

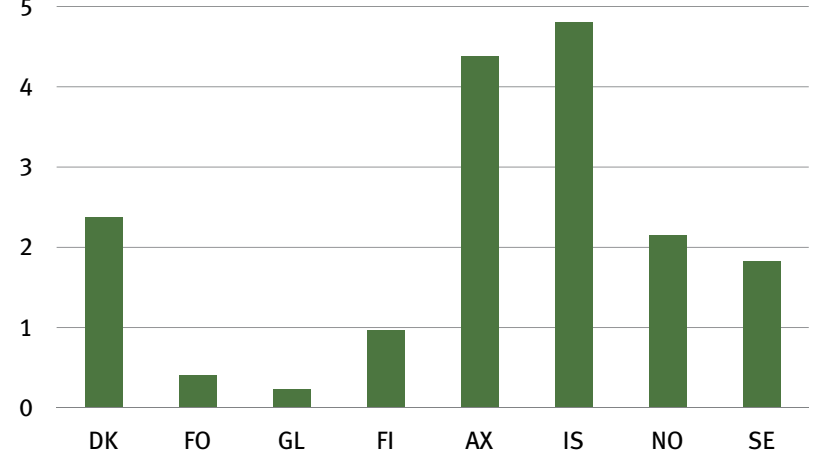

$\square$ Cult16 and Popu02 


\section{The Economy}

Economic key figures 2013

$\begin{array}{llllll}\text { DK } & \text { FI } & \text { IS } & \text { NO } & \text { SE } & \text { EA17 }\end{array}$

Per cent of GDP

Balance of payments

$\begin{array}{lllll}7.8 & -1.0 & 3.9 & 11.2 & 6.4\end{array}$

Foreign asssets, net,

31 Dec. 2013

$\begin{array}{rrrrrr}40.0 & 46.0 & -422.0 & 134.0 & . . & . . \\ -0.7 & -2.5 & -3.3 & 13.2 & -1.0 & -3.0\end{array}$

Public sector surplus

Public debt,

31 Dec. 2013

$\begin{array}{lllllll}48.6 & 60.7 & . . & . . & 41.7 & 95.2\end{array}$

Percent

Unemployment

$\begin{array}{llllll}7.0 & 8.2 & 5.4 & 3.5 & 8.0 & 12.0\end{array}$

Interest rate

$\begin{array}{llllll}1.7 & 1.9 & 5.8 & 2.6 & 2.1 & 3.0\end{array}$

Percentage change

2012-2013

Inflation

$\begin{array}{rrrrrr}0.5 & 2.2 & 4.1 & 2.0 & 0.4 & 1.4 \\ 0.4 & -1.4 & 3.3 & 0.6 & 1.6 & -0.4 \\ 20.0 & 16.4 & 19.6 & 14.0 & 17.1 & . .\end{array}$

Economic growth

Share price index

$-3.0$

$\begin{array}{lll}2.4 & -3.9 & 1.0\end{array}$

exchange rate index

$\begin{array}{llll}. . & 2.4 & -3.9 & 1.0\end{array}$

Key01. Harmonized unemployment figures. EA17: The 17 EU member states that had adopted the euro currency as their sole legal tender by 1 January 2013. 


\section{House price index}
Index $2000=100$

270

250

230

210

190

170

150

130

110

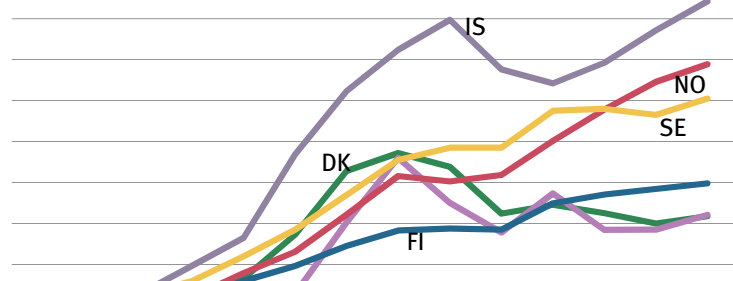

90

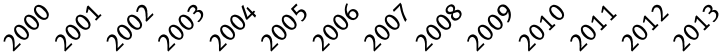

马Pric05

\section{Foreign direct investment - inwards}

Per cent of GDP

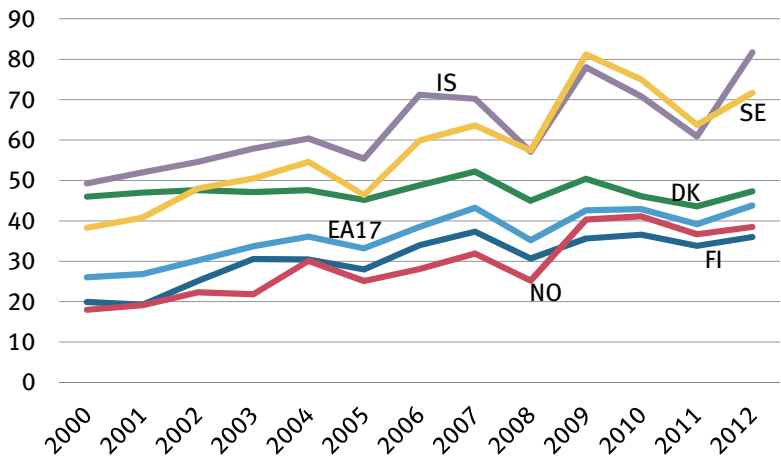

Fodi01. EA17: The 17 EU member states that had adopted the euro currency as their sole legal tender by 1 January 2013. 


\section{The Economy}

Gross domestic product per person in euro (PPS)

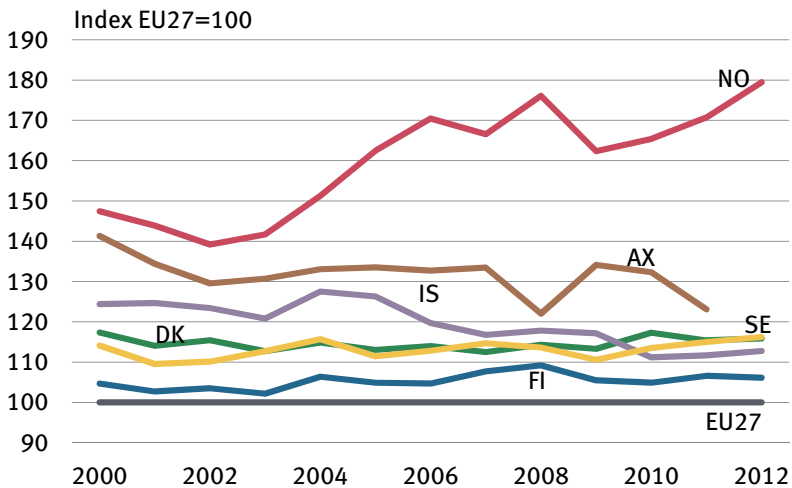

Naac04. EU27: The 27 EU member states as per 1 January 2013. PPS: Purchasing Power Standards which eliminates the differences in price levels between the countries.

\section{Gross domestic product, real annual growth}

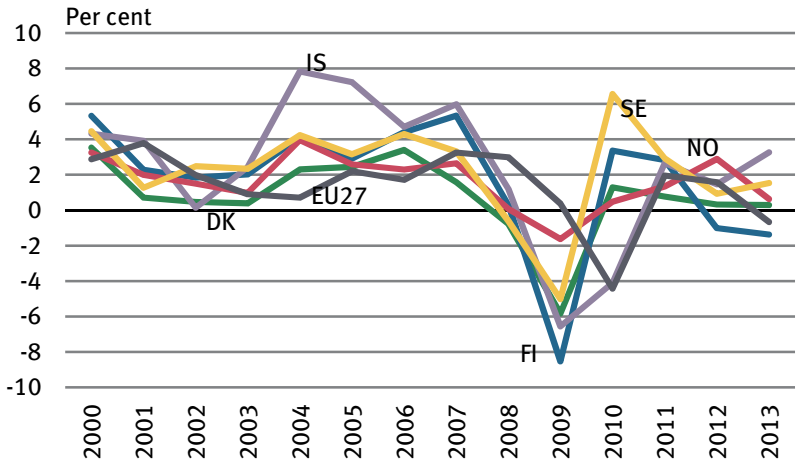

Naac01 and Key01. EA17: The 17 EU member states that had adopted the euro currencv as their sole leaal tender bv 1 lanuarv 2013. 
Nordic exports and imports in per cent of total export 2012

\begin{tabular}{|c|c|c|c|c|c|}
\hline Per cent & DK & FI & IS & NO & SE \\
\hline \multicolumn{6}{|l|}{ Exports to: } \\
\hline Total & 100.0 & 100.0 & 100.0 & 100.0 & 100.0 \\
\hline Nordic countries & 23.5 & 16.0 & 9.7 & 12.6 & 23.4 \\
\hline EU27 (excl. DK, Fl, SE) & 47.4 & 40.5 & 70.1 & 69.3 & 43.7 \\
\hline Other Europe & 2.3 & 3.7 & 2.7 & 1.4 & 3.1 \\
\hline USA and Canada & 7.5 & 7.4 & 5.1 & 5.5 & 7.2 \\
\hline Other America & 1.5 & 2.3 & 0.5 & 0.7 & 1.6 \\
\hline BRIC & 6.0 & 16.7 & 5.3 & 3.2 & 7.2 \\
\hline Japan & 2.0 & 1.9 & 2.1 & 0.9 & 1.5 \\
\hline DAEs & 3.7 & 3.3 & 1.0 & 4.3 & 2.9 \\
\hline Oceania & 1.2 & 1.3 & 0.2 & 0.3 & 1.4 \\
\hline Rest of the world & 4.7 & 7.0 & 3.5 & 1.8 & 8.1 \\
\hline \multicolumn{6}{|l|}{ Imports from: } \\
\hline Total & 100.0 & 100.0 & 100.0 & 100.0 & 100.0 \\
\hline Nordic countries & 22.5 & 21.1 & 27.5 & 22.9 & 22.1 \\
\hline EU27 (excl. DK, FI, SE) & 55.8 & 44.9 & 33.6 & 41.6 & 53.7 \\
\hline Other Europe & 2.2 & 1.5 & 2.5 & 2.9 & 1.7 \\
\hline USA and Canada & 3.0 & 2.8 & 11.2 & 8.3 & 3.6 \\
\hline Other America & 1.4 & 1.3 & 3.2 & 1.3 & 0.9 \\
\hline BRIC & 9.1 & 23.5 & 15.3 & 13.6 & 11.4 \\
\hline Japan & 0.5 & 0.5 & 1.5 & 2.3 & 1.4 \\
\hline DAEs & 2.0 & 1.9 & 3.1 & 3.8 & 2.4 \\
\hline Oceania & 0.3 & 0.3 & 0.1 & 0.2 & 0.2 \\
\hline Rest of the world & 3.3 & 2.2 & 1.9 & 3.0 & 2.6 \\
\hline
\end{tabular}

Fotr49. EU27: The 27 EU member states as per 1 January 2013. BRIC = Brazil, Russia, India and China. DAE-countries (Dynamic Asian Economies) = Hong Kong, Malaysia, Singapore, South Korea, Taiwan and Thailand. 
Foreign trade

Imports and exports to and from other Nordic countries 2012

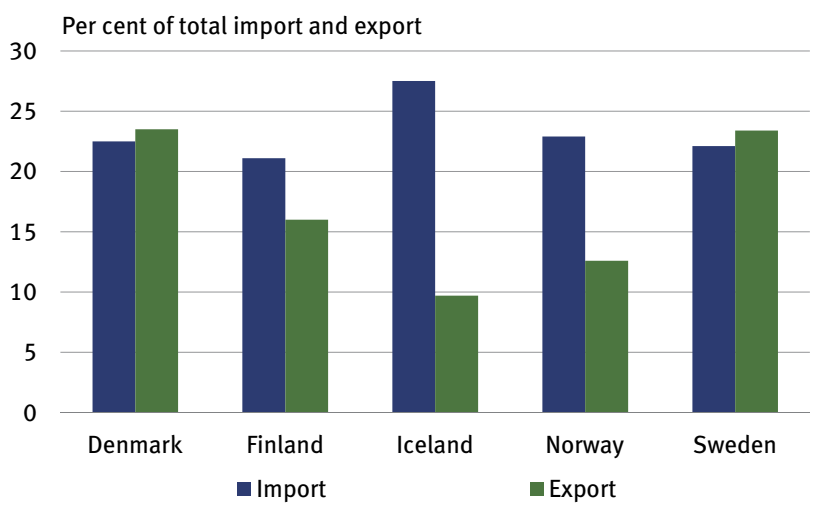

ㅁotr49

\section{Trade balance of goods}

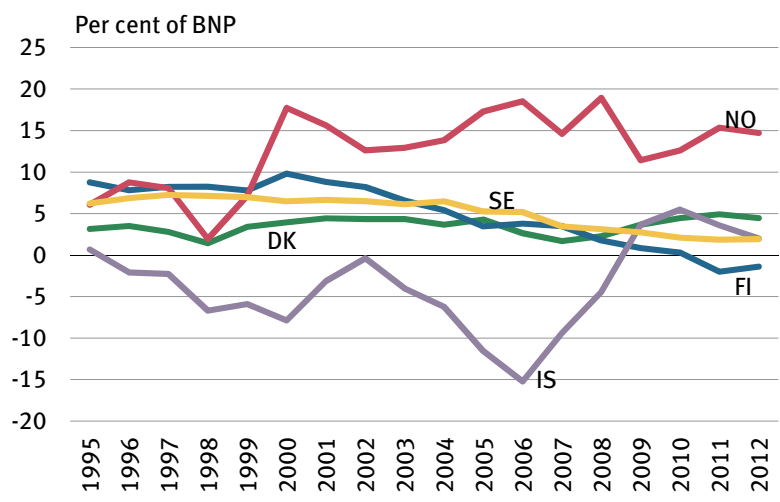

Fotr49 and Naac01 
Consumer price index 2013

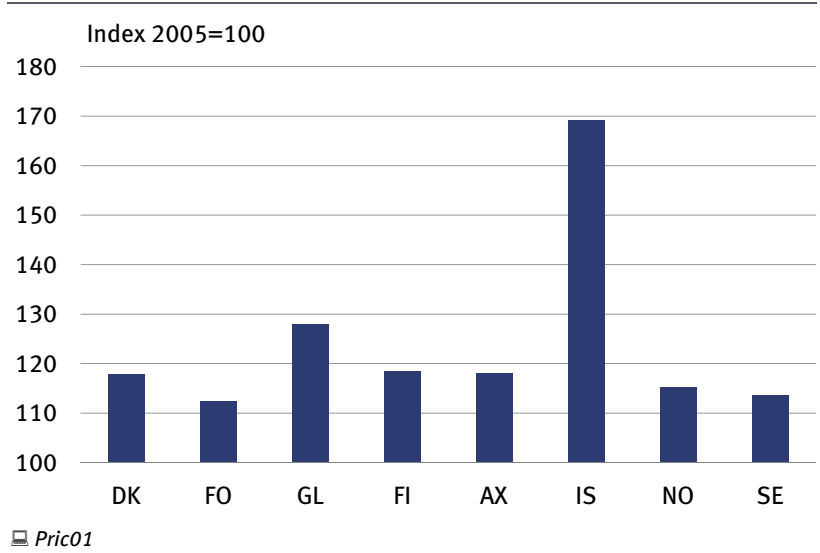

Price levels 2013

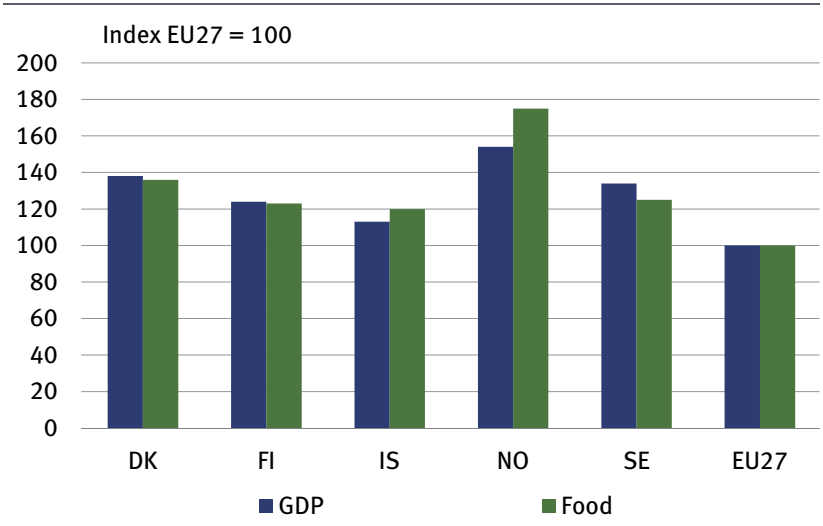

D Pric03. EU27: The 27 EU member states as per 1 January 2013. 


\section{Public finance and prices}

\section{General government expenditure by purpose 2012}

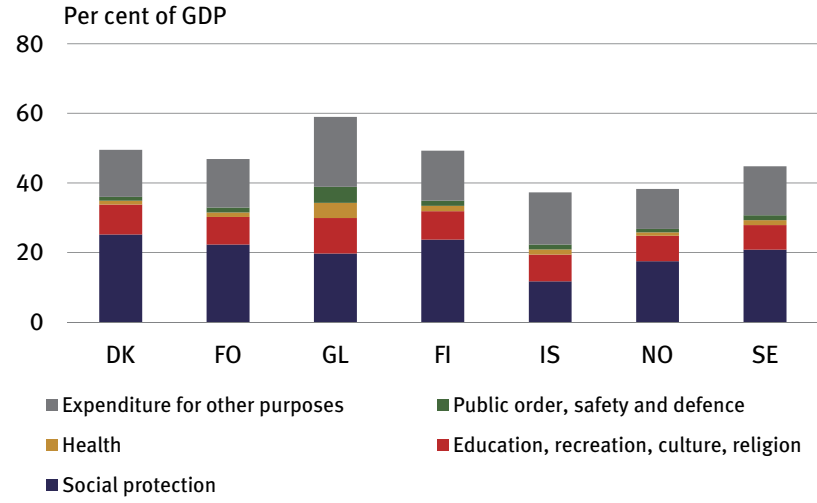

Pubs13. FO: 2011 data.

\section{General government gross debt}

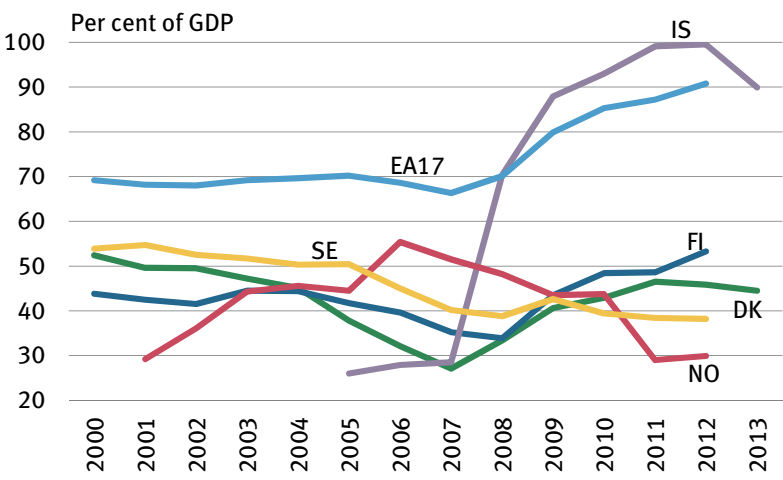

ㅁ Pubs14. EA17: The 17 EU member states that had adopted the euro currency as their sole legal tender by 1 January 2013. 


\section{Share prices}

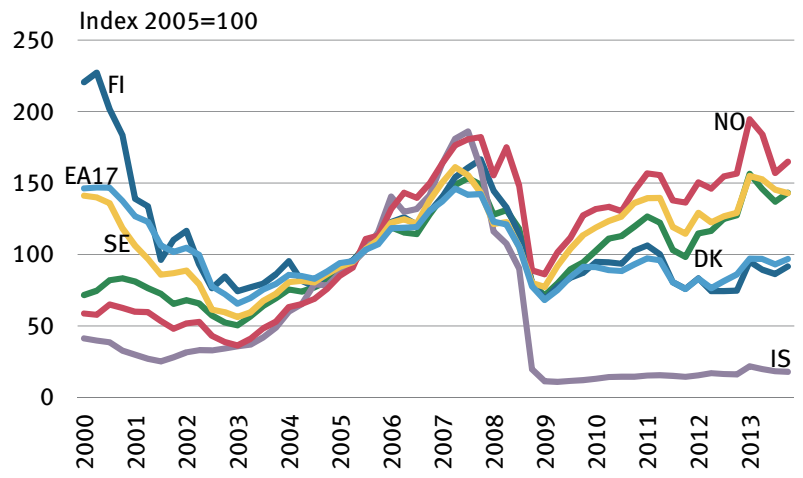

Shar01. EA17: The 17 EU member states that had adopted the euro currency as their sole legal tender by 1 January 2013.

\section{Government aid to developing countries}

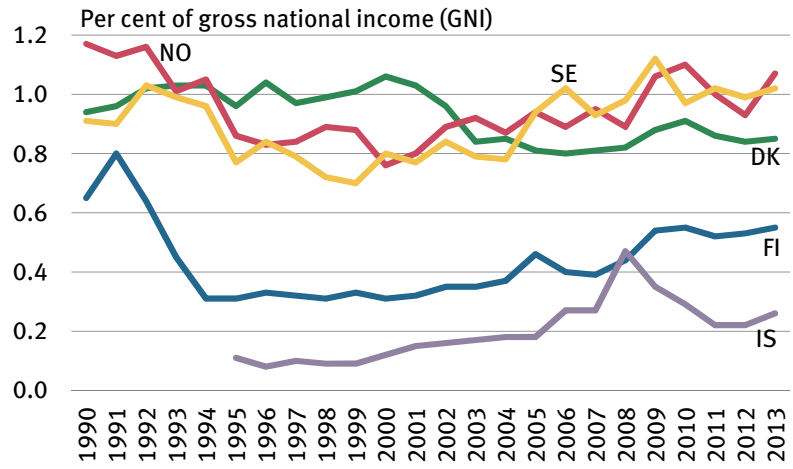

D Ofde02 
$R \& D$ expenditure by performing sector 2012

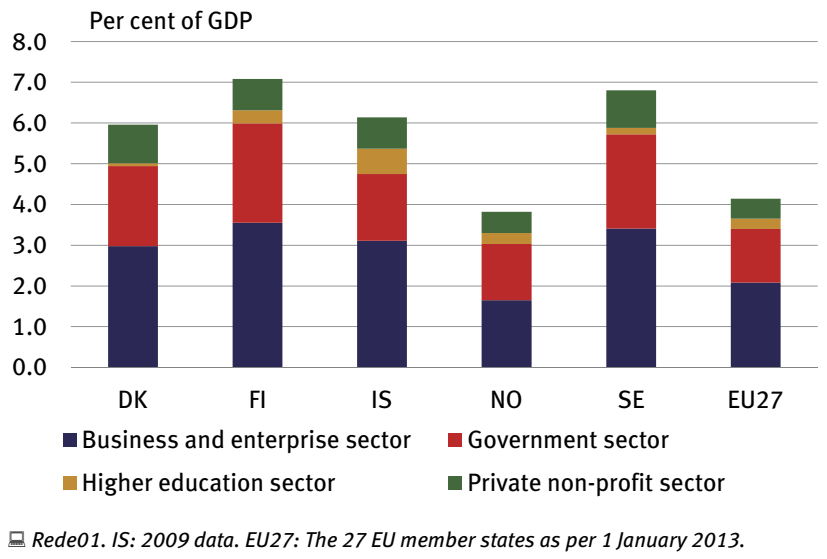

\section{Patents 2013}

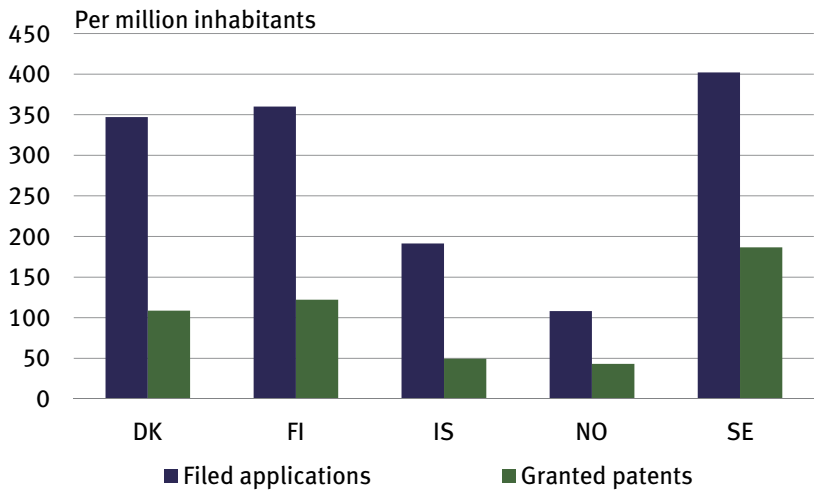


PhD graduates 2013

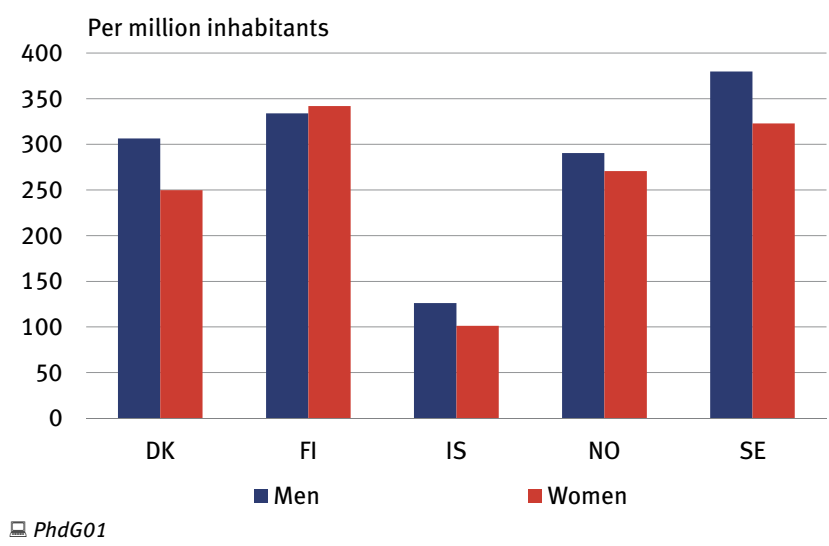

Internet purchases by individuals

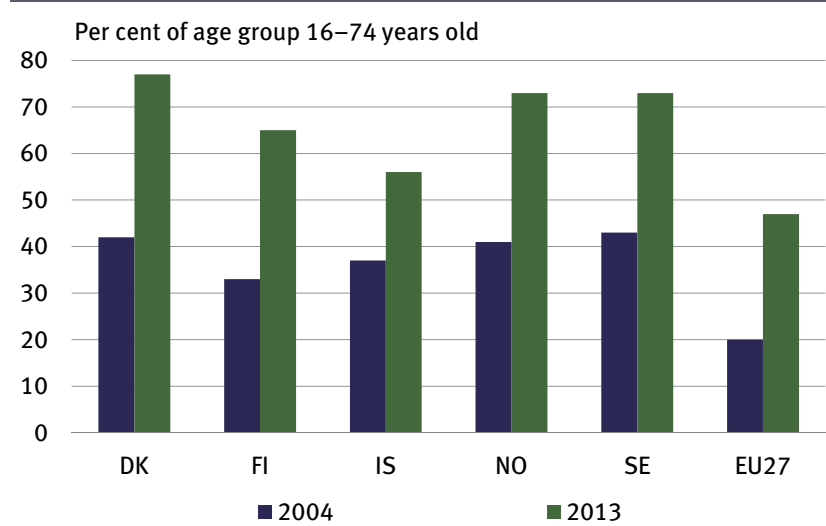

$\square$ Info13. EU27: The 27 EU member states as per 1 January 2013. EU27: 2004 data refers to 2009 data. 
Interaction of individuals with public authorities via the Internet 2013
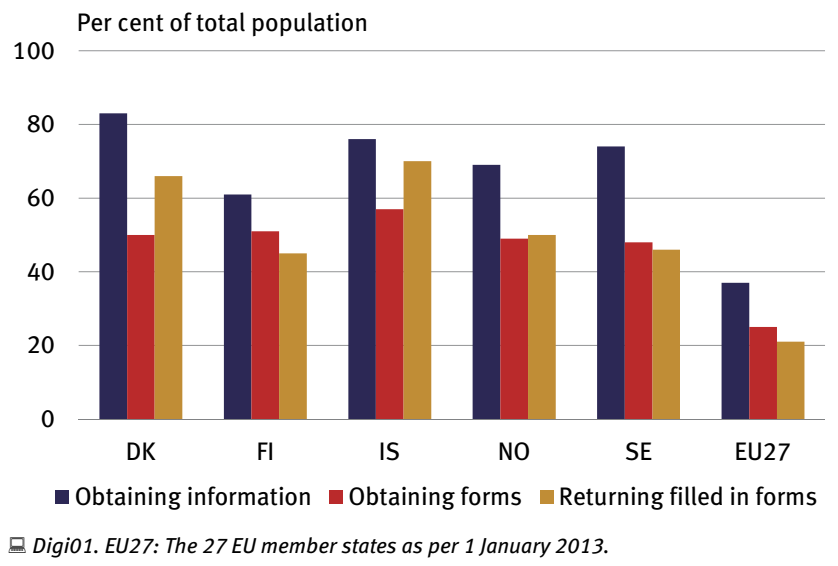

Interaction of enterprises with public authorities via the Internet 2013

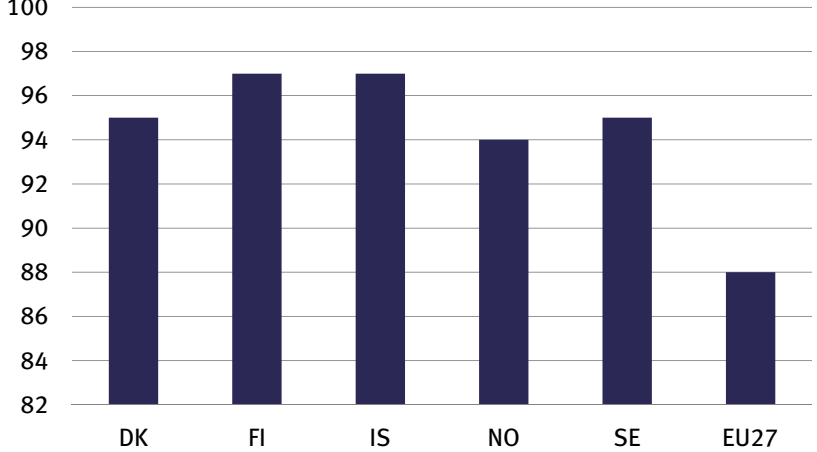

Digi02. EU27: The 27 EU member states as per 1 January 2013. 
Order your Nordic Statistical Yearbook 2014 on www.norden.org/nordpub 


\section{norden}

Nordic Council of Ministers

Ved Stranden 18

DK-1061 Copenhagen K

www.norden.org

This booklet presents an interesting selection of basic data on socioeconomic conditions and cultural affairs in the entire Nordic region. The figures are excerpts from the Nordic Statistical Yearbook 2014.

ISBN 978-92-893-3796-0 (PRINT) ISBN 978-92-893-3818-9 (PDF) ISSN 0908-4339 http://dx.doi.org/10.6027/ANP2014-752 ANP 2014:752 